\title{
Profiling Cloud Liquid Water by Combining Active and Passive Microwave Measurements with Cloud Model Statistics
}

\author{
U. Löhnert, S. CRewell, AND C. Simmer \\ Meteorological Institute, University of Bonn, Bonn, Germany
}

A. MACKE

Institute for Marine Research, Kiel, Germany

(Manuscript received 13 July 2000, in final form 28 November 2000)

\section{ABSTRACT}

\begin{abstract}
A method for combining ground-based passive microwave radiometer retrievals of integrated liquid water (LWP), radar reflectivity profiles $(Z)$, and statistics of a cloud model is proposed for deriving cloud liquid water profiles (LWC). A dynamic cloud model is used to determine Z-LWC relations and their errors as functions of height above cloud base. The cloud model is also used to develop an LWP algorithm based on simulations of brightness temperatures of a $20-30-\mathrm{GHz}$ radiometer. For the retrieval of $\mathrm{LWC}$, the radar determined $Z$ profile, the passive microwave retrieved LWP, and a model climatology are combined by an inverse error covariance weighting method. Model studies indicate that LWC retrievals with this method result in rms errors that are about $10 \%-20 \%$ smaller in comparison to a conventional LWC algorithm, which constrains the LWC profile exactly to the measured LWP. According to the new algorithm, errors in the range of $30 \%-60 \%$ are to be anticipated when profiling LWC. The algorithm is applied to a time series measurement of a stratocumulus layer at GKSS in Geesthacht, Germany. The GKSS 95-GHz cloud radar, a 20-30-GHz microwave radiometer, and a laser ceilometer were collocated within a 5-m radius and operated continuously during the measurement period. The laser ceilometer was used to confirm the presence of drizzle-sized drops.
\end{abstract}

\section{Introduction}

Clouds play a very important role within the hydrological cycle. The distributions of hydrometeor phase and size within clouds are important factors in the development of precipitation. Thus, the predictability of precipitation in numerical weather forecast models also depends on the correct description of liquid water content of developing clouds. The parameterization schemes used in state-of-the-art weather forecast models have large uncertainties and are not capable of describing actual cloud dynamics on scales that are significant for the formation of convection. One reason for this deficiency is that up to now, no appropriate datasets of microphysical cloud variables have been recorded due to restrictions in measurement capabilities. In the past, sporadic in situ aircraft measurements have been the only reliable method. Ground-based passive microwave measurements have proven to be quite accurate for determining integrated cloud liquid water (LWP; Westwater 1978; Peter and Kämpfer 1992), but retrieving the vertical distribution of cloud liquid water (LWC)

Corresponding author address: Ulrich Löhnert, Meteorologisches Institut Universität Bonn, Auf dem Hügel 20, 53121 Bonn, Germany. E-mail: uloeh@uni-bonn.de from brightness temperature (TB) measurements is very uncertain because the problem is underdetermined. A possibility is to combine active and passive microwave components to infer LWC profile information. In the last 5 to 10 years, active and passive ground-based microwave technologies have been developed for monitoring clouds in a quantitative way (Solheim et al. 1998; Clothiaux et al. 1995). Modern cloud radars have sufficiently high sensitivities to quantify radar reflectivity $(Z)$, Doppler velocity, and polarimetric qualities of clouds. High spectral, spatial, and temporal resolution allow the development of new techniques, which can help to quantify cloud microphysics at finer scales than before. In this study the Forschungszentrum Geesthacht GmbH (GKSS) 95-GHz radar was used together with a radiometer suited to measure the liquid water path with an accuracy of $10 \%-30 \%$. The combination of both instruments is necessary to constrain the inherent ambiguity of the radar measurements due to the variations of the cloud drop size distribution (DSD).

In the idealized case of Rayleigh backscattering, $Z$ is equal to the sixth moment of the cloud droplet distribution. Since LWC is proportional to the third moment of the DSD, varying DSDs cause large variations in the relationship between $Z$ and LWC. For example, if a 
TABLE 1. Mean values (Mean) and standard deviations (Std) of the modeled LWC $\left(\mathrm{g} \mathrm{m}^{-3}\right)$ in $625 \mathrm{~m}$ above cloud base, and Bias and rms errors of the Z-LWC relation with Bias(corr) and rms(corr) denoting the bias corrected version of the $Z$-LWC relation. All errors as functions of the different dBZ classes (Fig. 3).

\begin{tabular}{lccccrc}
\hline \hline dBZ Class & Mean & Std & Bias & Rms & \multicolumn{1}{c}{$\begin{array}{c}\text { Bias } \\
\text { (corr) }\end{array}$} & $\begin{array}{c}\text { Rms } \\
\text { (corr) }\end{array}$ \\
\hline Class A & 0.063 & 0.033 & -0.018 & 0.032 & 0.005 & 0.027 \\
Class B & 0.219 & 0.134 & -0.106 & 0.148 & -0.038 & 0.094 \\
Class C & 0.551 & 0.238 & -0.255 & 0.341 & -0.163 & 0.279 \\
Class D & 0.633 & 0.300 & 0.316 & 0.526 & 0.025 & 0.298 \\
Class E & 0.611 & 0.325 & 1.839 & 2.005 & 0.639 & 0.736 \\
All classes & 0.264 & 0.238 & -0.066 & 0.325 & -0.047 & 0.150 \\
\hline
\end{tabular}

volume of $1 \mathrm{~m}^{3}$ contains $0.1 \mathrm{~g}$ of liquid water and only droplets $5 \mu \mathrm{m}$ in radius, the reflectivity will be 8 times smaller than if the volume consists only of droplets 10 $\mu \mathrm{m}$ in radius. A very challenging problem in deriving cloud liquid water is the presence of drizzle drops (50$400 \mu \mathrm{m}$ in radius), which evaporate on their way to the ground and hence cannot be perceived as precipitation on the ground. These droplets frequently have high contributions to $Z$ but do not necessarily contain considerable amounts of LWC (Fox and Illingworth 1997a).

In past years many attemps have been made to relate cloud radar reflectivity factor measurements to microphysical cloud properties such as liquid water content, effective radius $\left(r_{\text {eff }}\right)$, or total number concentration $\left(N_{\text {tot }}\right)$. Sauvageot and Omar (1987) developed a universal Z-LWC relationship for non- or very weakly precipitating cumulus clouds. In situ aircraft measurements of drop size distribution were used to calculate $Z$. Here an upper limit of $-15 \mathrm{dBZ}$ was set to the validity of the relationship, because no clear correlation was found for higher reflectivities. This was interpreted as the limit between nonprecipitating and precipitating clouds. Fox and Illingworth (1997b) developed empirical Z-LWC relationships for stratocumulus clouds also based on in situ aircraft measurements. They proposed to distinguish between clouds with and without occasional drizzle according to the form of the vertical $Z$ profile. Errors are estimated to be $50 \%$ for a single retrieval of LWC for a nondrizzle cloud. Liao and Sassen (1994) used the output of a one-dimensional diffusional growth cloud model to simulate radar reflectivities from predicted droplet spectra. They found that the $Z-L W C$ relationship was mainly dependent on the activated cloud condensation nuclei $(\mathrm{CCN})$ concentration, which is equal to $N_{\text {tot }}$ in their model. In comparison with empirical based relationships for cumulus and stratocumulus clouds the best agreement was found for $N_{\text {tot }}$ of about $100 \mathrm{~cm}^{-3}$. Vivekanandan et al. (1997) used a radar-radiometer combination to derive $Z$-LWC relationships. They used a gamma function to describe the relationships between $N_{\text {tot }}$, LWC, Z, and the median diameter $D_{m}$. In a first step the radiometer derived LWP was used to determine a constant $D_{m}$ profile as a first guess. Then equations for $Z$ and LWC were iterated in $D_{m}$ and $N_{\text {tot }}$ to find an
LWC profile corresponding best to the measured LWP. The upper value for $D_{m}$ was set to $100 \mu \mathrm{m}$. Frisch et al. (1995) have developed a combined radar-radiometer LWC profile retrieval algorithm for stratus clouds, where the retrieved LWC is constrained exactly to the observed LWP. Typical DSD parameters for stratus clouds are incorporated.

Here a method is proposed to combine LWP, $Z$ profile, and an LWC model climatology in an optimal way to obtain the LWC profile. If $x_{i}$ denotes a scalar measurement with variance $\sigma_{i}^{2}$, then $m$ independent measurements can be combined to

$$
\hat{x}=\left(1 / \sum_{i=1}^{m} \sigma_{i}^{2}\right)^{-1}\left[\sum_{i=1}^{m}\left(x_{i} / \sigma_{i}^{2}\right)\right],
$$

$\hat{x}$ yielding an optimal estimator for $x$. This method is called inverse variance weighting or optimal estimation (OE; Rodgers 1976). For profile retrieval, $x_{i}$ will be a measurement vector and $\sigma_{i}^{2}$ the error covariance matrix. Since no direct measurements of LWC can be obtained, the inverse of a forward model function $\mathbf{F}$ is used to convert measurements $y_{i}$ into the space of $x$. Virtual measurements (e.g., a model climatology) of $x$ can be used as an $x_{i}$ as long as its variance (covariance matrix) $\sigma_{i}^{2}$ is known.

The paper is structured as follows. Section 2 describes the forward model function $\mathbf{F}$ and the model climatology. A dynamic cloud model with a resolved liquid cloud droplet spectrum is used. The model is initialized with radiosonde observations to account for realistic atmospheric conditions (sections $2 \mathrm{a}, \mathrm{b}$ ). Then the model output is used to derive both the $Z$-LWC relations and the LWP retrieval algorithm from simulated passive microwave measurements. The formulation for $\mathbf{F}$ is given in section 2e. Attenuation of the radar beam due to hydrometeors is taken into account and a method is proposed to correct for attenuation (sections $3 \mathrm{a}, \mathrm{b}$ ). Section 4 gives an introduction to the theory of optimal estimation (section 4a). The application to the combination radar-radiometer-cloud model is described in detail in section 4b. Here it is shown how the covariance matrices needed for a vector formulation of (1) are constructed. In section 5 the proposed retrieval method is applied to independent model data and compared with a standard retrieval method. The applications of the algorithm to real data is shown in section 6 . First, the instruments used to measure a time series of $Z$ profiles and LWP are described (sections 6a, b). Then, the characteristics of the day of measurement (section $6 \mathrm{c}$ ), and finally the LWC retrieval (section 6d) are discussed. The paper concludes with a summary and a description of further extensions of measurement combination using a highly sophisticated multichannel microwave radiometer. 


\section{Derivation of the forward model function and model climatology}

In this section the forward model function $\mathbf{F}$ is derived, which combines the $Z$-LWC relation and the retrieved LWP. Here, $\mathbf{F}$ will be of the general form

$$
\mathbf{y}=\mathbf{F}(\mathbf{L W C})
$$

with $\mathbf{y}$ denoting the measurement vector consisting of the $Z$ profile from the cloud radar and the corresponding LWP derived from the radiometer measurements. A 1.5dimensional time-dependent convective cloud model (Issig 1997) is used to simulate the dependencies between $Z$ and LWC. The model is also used to develop a linear retrieval for LWP from simulated passive microwave brightness temperatures. The mean of the modeled LWC is used as the cloud climatology. For initialization the model requires vertical profiles of temperature, pressure, and humidity. To include all kinds of weather conditions, $6 \mathrm{yr}$ of daily (1200 UTC) radiosonde data from the time period between 1984 and 1992 are used from the station Essen of the German Weather Service. The data are devided into three sets. The TRAIN (training) dataset encompasses the years 1984, 1986, and 1988. The corresponding model output data are used to derive the algorithms. The TEST (testing) dataset is comprised of the 1990 data, and the model statistics based on TEST are used to determine the covariance matrices. The 1992 dataset GENE (generalization) was used to evaluate the algorithms.

\section{a. Cloud model description}

The cloud model (Issig 1997) predicts liquid drop size spectra for 40 logarithmically scaled radius intervals with 250-m vertical resolution. Droplet sizes range from 1 to $10^{4} \mu \mathrm{m}$. For each droplet class a prognostic mass budget equation is solved including detailed microphysics such as diffusion, coagulation, and break up. Further prognostic equations yield vertical velocity, temperature, water vapor, and ice phase. All equations include a vertical advection term, a formulation for the entrainment, and a microphysical term. The vertical velocity equation additionally includes the buoyancy term. The entrainment term describes the interaction between the cloud and its surrounding environment. The ice phase is parameterized in bulk form according to van der Emde and Kahlig (1989) and is considered to consist of ice and graupel. In 1.5 dimensions the cloud is perceived as a cylinder with a radius $r_{c}$ and no variations in azimuth direction. Inside the cylinder the variables are height- and time-dependent, whereas the variables of the surrounding are maintained constant in a stable cloud-free atmosphere. Convection is initialized by realistic diabatic processes via a radiation module. A typical CCN spectrum for land conditions was chosen according to empirical results from Pruppacher and Klett (1997). At the ground, the initial total number concen- tration of $\mathrm{CCN}$ is set to $1000 \mathrm{~cm}^{-3}$ decreasing exponentially to $100 \mathrm{~cm}^{-3} 5 \mathrm{~km}$ above ground. Then the total number concentration of $\mathrm{CCN}$ is held constant up to the model top height at $10 \mathrm{~km}$.

\section{b. Model evaluation}

The radiosonde ascents from TRAIN, TEST, and GENE are used to initialize the cloud model. The prognostic variables are simulated in 40 heights. Results are recorded every $40 \mathrm{~s}$ for a duration of $2 \mathrm{~h}$ after initialization. For each time step, and in each height the sixth moment of the drop size distribution is calculated, which is equivalent to $Z$ in the Rayleigh approximation. To reduce the data volume, only those cases are considered where two time steps differ by more than $30 \mathrm{~g} \mathrm{~m}^{-2}$ in LWP. These time steps are examined in each height for the presence of clouds, which are assumed to be present if a threshold of $0.005 \mathrm{~g} \mathrm{~m}^{-3}$ is exceeded. To exclude raining clouds, profiles with droplets larger than 400 $\mu \mathrm{m}$ in radius contributing more than $-40 \mathrm{dBZ}$ are excluded. Thus, occasional drizzle cases produced by the model are included in the statistics. These are here defined by a $\mathrm{dBZ}$ contribution in the radius interval [50, 400] $\mu \mathrm{m}$ being larger than $-40 \mathrm{dBZ}$. After a model time of $4000 \mathrm{~s}$ about $10 \%$ of the evaluated cases show drizzle, which, however, only contributes about $0.5 \%-$ $4 \%$ to the total LWP. Following reasons of simplicity, the ice phase is neglected in all cases and does not contribute to the calculated radar reflectivity. Thus, the algorithm is only applicable to cases free of ice. Figure 1 shows the mean development of the simulated clouds in time in terms of LWP. As expected for these nonprecipitating clouds, the maximum value of LWP is reached in a very early phase of cloud development, corresponding to the maximum values of upward vertical velocity. Figure 2 indicates the mean model LWC profiles and their standard deviations of four different vertical extensions.

An essential demand the model should meet is a correct description of the natural mean and variability of the LWC profile. This is important for the LWC algorithm presented in section 4 since the mean LWC profile and its variance is used as a week constraint. Due to the lack of long-term in situ LWC profile observations, a comparison between cloud model LWC and an empirically modified adiabatic method (Karstens et al. 1994) for determining LWC in defined cloud boundaries was carried out. Results show that the mean LWC of the modified adiabatic method lies within one standard variation of the mean model profiles (Fig. 2) for clouds with vertical extensions from 500 to $1500 \mathrm{~m}$. Additionally we checked LWC profiles derived from single in situ DSD measurements and found good agreement with the model climatology. These results indicate that the cloud model decribes the LWC climatology fairly well. 

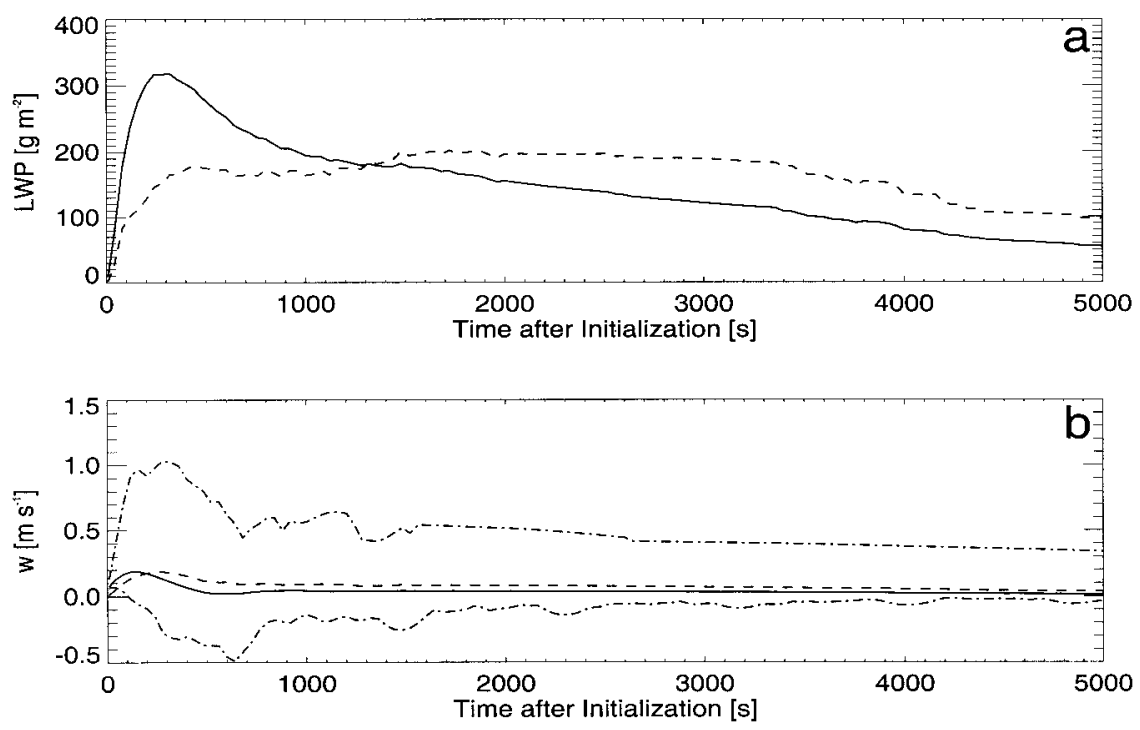

FIG. 1. Mean time series of cloud development. Top: mean LWP (solid line) and LWP standard deviation (dashed). Bottom: mean vertical velocity $w$ (solid), standard deviation of $w$ (dashed) and, minimum and maximum values of $w$ (dashed-dotted), respectively.

\section{c. Z-LWC relations}

The vertical coordinate used to describe the dependencies of LWC and $Z$ with height is height above cloud base. The typical form relating $Z$ to LWC is a powerlaw equation of the form

$$
Z=a \mathrm{LWC}^{b}
$$

(e.g., Sauvageot and Omar 1987; Fox and Illingworth 1997a). The errors when estimating LWC with this
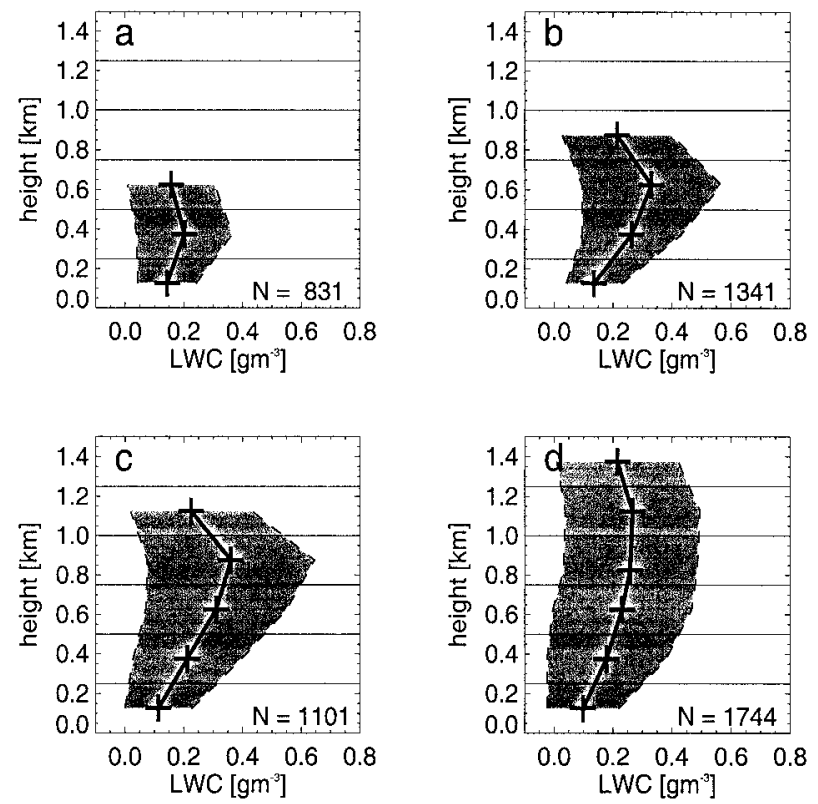

FIG. 2. Mean LWC profiles (thick line) and the corresponding 1$\sigma$ range (gray shaded). (a)-(d) show LWC profiles for different cloud vertical extension; $N$ depicts the number of profiles used. equation can be in the range of 1 order of magnitude or more due to variations in the droplet spectrum. To illustrate the sensitivity of (3), the lognormal size distribution, often used to characterize cloud and rain distributions (e.g., Feingold et al. 1997), is used:

$$
N(\ln r)=\frac{N_{\text {tot }}}{\sqrt{2 \pi} \sigma_{l}} \exp \left(-\frac{\left(\ln r-\ln r_{0}\right)^{2}}{2 \sigma_{l}^{2}}\right) .
$$

The three parameters describing this DSD are $\sigma_{l}(\log$ arithmic width), $r_{0}$ (modal radius), and $N_{\text {tot }}$ (total number of drops per unit volume). It can be shown that (3) will be exactly satisfied if two of the three DSD describing parameters in (4) are held constant (Erkelens et al. 1998). In the three possible cases, the exponent $b$ will vary between 1 and 4 giving rise to a wide range of variability. LWC derived from a typical cloud reflectivity value of $-35 \mathrm{dBZ}$ will vary in one order of magnitude if the exponent $b$ is varied between 1 and 4 .

To determine the coefficients $a$ and $b$ from the model simulations (3) is logarithmized to

$$
\mathrm{dBZ}=10 \log a+10 b \operatorname{logLWC},
$$

with $\mathrm{dBZ}=10 \log \left(Z / Z_{0}\right)$, whereby $Z_{0}$ is given by 1 $\mathrm{mm}^{6} \mathrm{~m}^{-3}$. Subsequently, in each 250 -m height interval above cloud base, a weighted linear regression of (5) is performed. This means that before the regression is performed, each (dBZ, LWC) pair is classified into one of five $\mathrm{dBZ}$ classes (A-E). The regression is performed with an equal number of (dBZ, LWC) pairs from each class. Every pair of the class with the minimal number $N_{\min }$ of pairs is taken into the regression, whereas $N_{\min }$ (dBZ, LWC) pairs from the other classes are randomly chosen. To perform a representative regression $N_{\text {min }}$ should at least be equal to 20 . This condition could be 

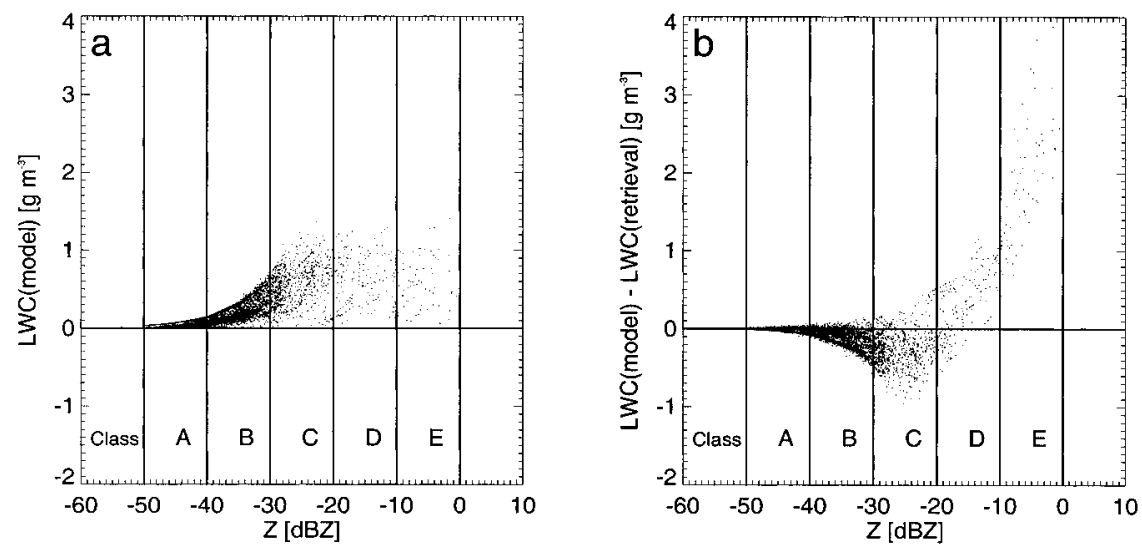

FIG. 3. Scatterplots in $625 \mathrm{~m}$ above cloud base. (a) Z-LWC dependency and (b) retrieval accuracy with a derived $Z$-LWC relation. The vertical lines depict the different $\mathrm{dB} Z$ classes.

satisfied up to a height of $1500 \mathrm{~m}$ above cloud base for the cloud-model results obtained from the TRAIN dataset. Performing a linear regression with (5) leads to a much stronger weighting of very small values of LWC than larger values due to minimizing the error between $\mathrm{dBZ}$ and $\log (\mathrm{LWC})$. This results in an almost bias-free determination of LWC in $\mathrm{dBZ}$ class A (Fig. 3, Table 1) but increasing absolute biases in classes of higher dBZ. The bias in class $\mathrm{E}$ is particularly high. This can be explained by large droplets contributing to high reflectivities but not increasing the mean LWC to values significantly larger than those in the classes $\mathrm{C}$ and $\mathrm{D}$. This behavior of LWC over- and underestimations is used to derive a bias corrected Z-LWC relation (Table 1).

Values of the coefficient $b$ derived from the cloud model are in the range of 1.85 to 2.58 , whereas $a$ ranges from 0.02 to 0.16 . These coefficients are in good agreement with values derived by Sauvageot and Omar (1987) from aircraft measurements of DSDs in a variety of cumulus, stratocumulus and stratus clouds. The coefficients $a$ and $b$ needed for (3) were created using the TRAIN dataset. The coefficients are derived as functions of height above cloud base and cloud thickness. The rms errors when applying the derived coefficients to the independent TEST dataset are used to derive the covariance matrices in section $4 \mathrm{~b}$.

\section{d. LWP retrieval}

In this work a simple statistical algorithm is developed to retrieve LWP from TB's measured at 21.3, 23.8, and $30.7 \mathrm{GHz}$. Water vapor and liquid water absorption are dominant at these frequencies with the latter being proportional to the third moment of the DSD. Generally a 20-30-GHz combination is necessary to retrieve LWP to separate the emission effects of water vapor (absorption line at $22.235 \mathrm{GHz}$ ) and cloud liquid (atmospheric window in the $30-\mathrm{GHz}$ region). The algorithm relies on the same cloud-model profiles of temperature, pressure, humidity, and cloud liquid water as used to obtain the statistics of the Z-LWC relationship (section $2 c)$. The TRAIN dataset is used to derive a linear relationship between the measured brightness temperatures and LWP. A simple orthogonal regression is performed to derive the coefficients $l_{0}, l_{1}, l_{2}, l_{3}$ of the relation

$$
\mathrm{LWP}=l_{0}+l_{1} \mathrm{~TB}_{21}+l_{2} \mathrm{~TB}_{23}+l_{3} \mathrm{~TB}_{31},
$$

with $l_{0}=-0.267, l_{1}=0.022, l_{2}=-0.029$, and $l_{3}=$ 0.027 . Since LWP algorithms for passive microwave measurements tend to get very inaccurate when LWP is larger than $1.0 \mathrm{~kg} \mathrm{~m}^{-2}$ due to large fractions of rain water (Karstens et al. 1994), only those time steps were included in the statistics when LWP was less than 1.0 $\mathrm{kg} \mathrm{m}^{-2}$. To simulate the TBs from the cloud-model profiles, gaseous absorption $\left(\mathrm{H}_{2} \mathrm{O}, \mathrm{O}_{2}\right)$ is calculated according to Liebe et al. (1993), whereas absorption and scattering coefficients due to hydrometeors are calculated according to Lorenz-Mie theory. The forward radiative transfer is calculated using the microwave radiative transfer model (MWMOD; Simmer 1994), which solves the atmospheric radiative transfer equation (ARTE) with the "successive order of scattering" method. When performing the regression, the TBs are subject to Gaussian noise corresponding to the measurement error (section $5 \mathrm{~b}$ ) to simulate real conditions. This leads to a modeled relative error of about $10 \%$ in LWP. The errors when applying this algorithm to the TEST dataset are also included in the covariance matrices in section $4 b$.

\section{e. Formulation of the forward model function}

Now two conditions which the forward model function $\mathbf{F}$ should meet can be stated. The first $l$ elements of $\mathbf{F}$ (corresponding to $l$ height dependent $Z$-LWC relations) should fulfill

$$
\mathrm{dBZ}_{i}=10 \log a_{i}+b_{i} \log \mathrm{LWC}_{i},
$$

with $i$ denoting the height index $(i \in[1, l])$. Then as a 

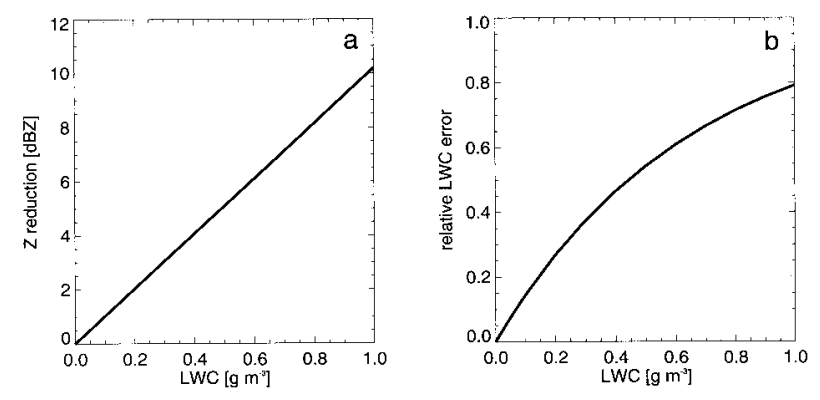

FIG. 4. Hydrometeor attenuation effects at $95 \mathrm{GHz}$ of a liquid water cloud of 1000-m vertical extension (constant LWC with height); (a) $\mathrm{dBZ}$ reduction (two-way attenuation) at cloud top assuming a cloud temperature of $0^{\circ} \mathrm{C}$, and (b) relative LWC error due to attenuation on a standard Z-LWC relation $(a=0.01, b=2)$.

second condition, the last element of $\mathbf{F}$ should constrain the retrieved (integrated) $\mathrm{LWC}_{i}$ measurements to the liquid water path as

$$
\mathrm{LWP}=\sum_{i=1}^{l}\left(\mathrm{LWC}_{i}\right) \Delta z
$$

where $\Delta z=250 \mathrm{~m}$ is the vertical model resolution. The measurement vector $\mathbf{y}$ is defined as ( $\mathbf{d B Z}$, LWP), where $\mathbf{d B Z}$ denotes the measured radar reflectivity vector and LWP the liquid water path derived from the TB measurements (6). If $\mathbf{F}$ in (2) is simplified to a linear problem by means of a Taylor series around a guessed $\mathbf{L W C} \mathbf{C}_{n}$ vector, then $\mathbf{y}$ can be written as

$$
\begin{aligned}
\mathbf{y}= & \mathbf{F}\left(\mathbf{L W C} \mathbf{C}_{n}\right)+\frac{\partial \mathbf{F}}{\partial \mathbf{L W C}}\left(\mathbf{L W C}-\mathbf{L W C}_{n}\right) \\
& +O\left(\mathbf{L W C}-\mathbf{L W C} \mathbf{C}_{n}\right)^{2}
\end{aligned}
$$

or, by redefining some of the symbols and omitting higher-order terms,

$$
\mathbf{y}=\mathbf{y}_{n}+\mathbf{K}_{n}\left(\mathbf{L W C}-\mathbf{L W C}_{n}\right)
$$

with $\mathbf{K}_{n}$ denoting the Jacobi matrix of the problem. Due to the errors in the LWP retrieval, errors in the Z-LWC relation, attenuation errors (section 3 ), and measurement errors, (10) will never be fulfilled exactly. This error can be minimized, however, by the OE method (section 4).

\section{Attenuation}

When $\mathrm{dBZ}$ profiles at $95 \mathrm{GHz}$ are interpreted to derive cloud microphysical properties, attenuation due to hydrometeors has to be accounted for.

\section{a. Simulation of attenuation}

Figure 4a shows hydrometeor attenuation effects at cloud top for a cloud consisting of liquid drops and a thickness of $1000 \mathrm{~m}$. Because of the temperature dependence of attenuation, the curve may vary within a range of $1 \mathrm{dBZ}$. This variability can be accounted for if, for example, the cloud-base temperature is inferred from an infrared radiometer. Since this type of measurement was not given during the measured time series, all clouds are assumed to have a constant temperature of $0^{\circ} \mathrm{C}$. The total opacity of the cloud was calculated using the Rayleigh approximation. According to Fig. $4 \mathrm{a}$, a cloud containing $0.4 \mathrm{~kg} \mathrm{~m}^{-2}$ of liquid water experiences a $\mathrm{dBZ}$ reduction of about $4 \mathrm{dBZ}$ at cloud top (two-way attenuation) causing a relative error of 50\% in LWC at cloud top (Fig. 4b).

\section{b. Correction for attenuation}

If absorption can be described by the Rayleigh approximation, $k$ is related linearly to LWC (Ulaby et al. 1981):

$$
k=c\left(T, \lambda_{0}\right) \mathrm{LWC},
$$

with $c$ a constant depending on the physical temperature $T$ of the cloud, and the radar wavelength $\lambda_{0}$. As before, all clouds are assumed to have a mean temperature of $0^{\circ} \mathrm{C}$. The total optical depth $\tau_{\text {cloud }}$ of a cloud layer is given by

$$
\tau_{\text {cloud }}=\int_{z_{\text {base }}}^{z_{\text {top }}} k(z) d z .
$$

The reflectivity factor $Z_{\text {top }}$, originating from cloud top, attenuated by the water cloud, will then be observed as

$$
Z_{\text {at }}=Z_{\text {top }} \exp \left(-2 \tau_{\text {cloud }}\right)
$$

at the radar site. With the assumption that $Z$ measured in the lowest cloud level is not attenuated, $k$ can be calculated using (11) and (3)

$$
k\left(z_{\text {base }}\right)=c\left(T, \lambda_{0}\right)\left[Z\left(z_{\text {base }}\right) / a\right]^{1 / b} .
$$

Now the optical depth of the lowest layer can be calculated and, consequently, the corrected reflectivity of the second layer can be computed by using an equation of the form of (13). This process can be repeated up to the cloud top in order to provide the complete $Z$ profile corrected for attenuation. To simulate measured radar data, the model results of $Z$ of the TEST dataset are analytically attenuated with an exponential relation as in (13). The term $\tau_{\text {cloud }}$ can be calculated using the model output data. Then the attenuated $Z$ profiles are "de"attenuated by using the method described above. The rms errors of the deattenuated $Z$ values are at maximum about $3 \mathrm{dBZ}$ at cloud-top height for clouds with vertical extension of $1500 \mathrm{~m}$. Most of this error is due to the bias in the region of $\mathrm{dBZ}$ values larger than -20 where errors of up to $-15 \mathrm{dBZ}$ are possible. In this region of larger optical depths the attenuation correction can become unstable and should be regarded with care. For $Z$ $\leq-20 \mathrm{dBZ}$ the derived errors can then be interpreted as measurement errors and are included in the covariance matrices derived in section 4 . 

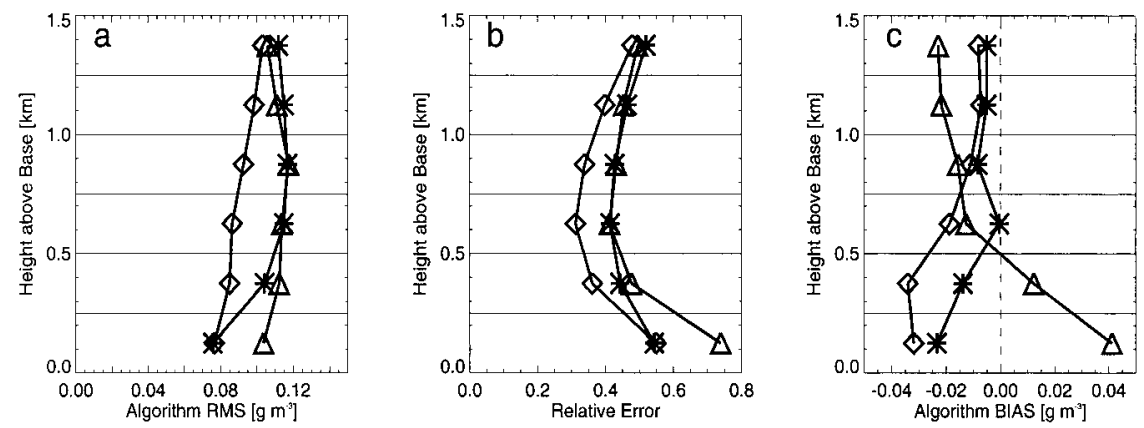

FIG. 5. Errors (rms, relative, bias) of different LWC retrievals in dependence of height above cloud base. Diamonds: optimal estimation with inclusion of the a priori profile; stars: optimal estimation without the a priori profile; triangles: method according to Frisch et al. (1998).

\section{Retrieval technique}

In this section the retrieval technique for LWC profiles is presented. First, the theory of optimal estimation is introduced following Rodgers (1976). The OE method is often used to retrieve temperature or humidity profiles from multispectral remote sensors (e.g., Ma et al. 1999). Subsequently, it is shown how this technique can be adapted to retrieve LWC profiles from a combination of a model climatology, Z measurements, and LWP measurements.

\section{a. Optimal estimation}

One major problem of remote sensing is the inversion of the atmospheric radiative transfer equation. Although forward calculations of atmospheric radiative transfer (i.e., calculation of radiances from the known atmospheric state) can be performed quite accurately, the inversion (even of the linearized ARTE) is complicated because a continuous profile of an atmospheric quantity is desired, whereas only a limited number of often highly correlated observations is available. This leads to an underdetermined problem, meaning that an infinity of solutions exists. As a consequence, profiles which are to be retrieved have to be discretizised and combined with other independent measurements. If additional measurements are not available the measurement vector and its covariance can be combined with a so-called virtual measurement (e.g., the information content contained in the climatology of the desired parameter, here the so-called a priori information). This combination will decrease the number of degrees of freedom of the problem and lead to a solution partially constrained by the climatological mean. If there are $m$ independent measurements $\mathbf{y}_{i}$ with $i \in[1$, $m$ ] and $\mathbf{x}$ denoting the unknown profile, a linear version of the ARTE can be written as

$$
\mathbf{y}_{i}=\mathbf{K}_{i} \mathbf{x},
$$

where $\mathbf{K}_{i}$ denotes the linear forward function of radiative transfer. The most likely value of $\mathbf{x}$ given the measurements $\mathbf{y}_{i}$ can be calculated by maximizing the condi- tional probability function $\mathbf{P}\left(\mathbf{x} \mid \mathbf{y}_{1}, \mathbf{y}_{2}, \ldots, \mathbf{y}_{m}\right)$ with $\mathbf{P}(\mathbf{x})$ defined as

$$
\mathbf{P}(\mathbf{x})=\frac{1}{(2 \pi)^{m / 2} \operatorname{det}(\mathbf{S})^{1 / 2}} \exp \left[-\frac{1}{2}\left(\mathbf{x}-\mathbf{x}_{0}\right)^{\mathrm{T}} \mathbf{S}^{-1}\left(\mathbf{x}-\mathbf{x}_{0}\right)\right],
$$

with $\mathbf{x}_{0}$ denoting the expected value of $\mathbf{x}, E[\mathbf{x}]$ and $\mathbf{S}$ the covariance matrix $\mathbf{S}=E\left[\left(\mathbf{x}-\mathbf{x}_{0}\right)\left(\mathbf{x}-\mathbf{x}_{0}\right)^{\mathrm{T}}\right]$. Applying Bayes' theorem and maximization leads to the set of the optimal estimation equations yielding the optimal solution

$$
\hat{\mathbf{x}}=\left(\sum_{i=1}^{m} \mathbf{K}_{i}^{\mathrm{T}} \mathbf{S}_{i}^{-1} \mathbf{K}_{i}\right)^{-1}\left(\sum_{i=1}^{m} \mathbf{K}_{i}^{\mathrm{T}} \mathbf{S}_{i}^{-1} \mathbf{y}_{i}\right)
$$

and its covariance $\hat{\mathbf{S}}$

$$
\hat{\mathbf{S}}=\left(\sum_{i=1}^{m} \mathbf{K}_{i}^{\mathrm{T}} \mathbf{S}_{i}^{-1} \mathbf{K}_{i}\right)^{-1} .
$$

Here, $\mathbf{S}_{i}$ represents the covariance matrix of the measurement vector $\mathbf{y}_{i}$. If the error statistics are assumed to be Gaussian, $\hat{\mathbf{x}}$ corresponds both to the maximum likelihood and the expected value solution.

\section{b. Application to combined radar-radiometer measurements}

The radar equation valid for meteorological targets (Ulaby 1981) is also a type of ARTE. One can calculate the power backscattered to the radar receiver $P_{r}$ if transmitted power, antenna gain, wavelength, and the atmospheric composition (i.e., hydrometeors, gases, and temperature) are known:

$$
P_{r}=\frac{C}{R^{2}} \sigma_{v} \exp (-2 \tau)
$$

with $R$ denoting the range to the scattering volume, $\tau$ the opacity, and $\sigma_{v}$ the radar cross section of the scattering volume, the latter two resulting from the atmospheric composition. Here, $C$ is the radar constant and 
a function of transmitted power, antenna gain, and wavelength. If the backscattering process can be described in terms of Rayleigh scattering, and with the radar reflectivity factor defined as

$$
Z=\sum_{i} N\left(D_{i}\right) D_{i}^{6},
$$

with $N$ denoting the number of drops per unit volume and $D_{i}$ the cloud-drop diameter, $\sigma_{v}$ can be expressed as

$$
\sigma_{v}=\frac{64 \pi^{5}}{\lambda_{0}^{4}}|K|^{2} \sum_{i} N\left(D_{i}\right) D_{i}^{6}
$$

with $K$ a complex quantity dependent on the complex index of refraction of water. Combining (19), (20), and (21), one can write

$$
Z=\frac{P_{\mathrm{ra}}}{C^{\prime}} e^{(2 \tau)},
$$

with $C^{\prime}$ denoting a modified radar constant. The $R^{2}$ dependency is eliminated by multiplying (19) with $R^{2}$, which can be determined by the time difference between the transmitted and received radar pulse. Consequently, $P_{\mathrm{ra}}$ is the so-called range-adjusted power $P_{r} R^{2}$.

Equation (3) can also be regarded as a simplified ARTE. Together with LWP measurements from the microwave radiometer and an a priori climatology from the cloud model, the OE retrieval method can be applied. In the following the measurement vector $\mathbf{y}$ as described in (10) will now be denoted by $\mathbf{y}_{1}$. Further information is included by taking as a second measurement vector $\mathbf{y}_{2}$ as the mean model profile climatology of LWC derived from the TEST dataset. The inverse error covariance weighting method will lead to a solution for the LWC profile, which will satisfy the forward function $\mathbf{F}$ within its errors as stated in section 2e. Additionally, the LWC retrieval is constrained to the model climatology $\mathbf{y}_{2}$ taking into account its variance. Thus, the problem consists of two independent measurements that are to be combined. The radiative transfer model MWMOD (Simmer 1994) and the cloud model (Issig 1997) are used to derive the two covariance matrices $\mathbf{S}_{1}$ and $\mathbf{S}_{2}$ needed in our case. Covariance matrix $\mathbf{S}_{2}$ consists of the LWC covariances of the mean model profile derived from the TEST dataset. In this case, $\mathbf{K}_{2}$ will be the identity. Covariance matrix $\mathbf{S}_{1}$ is a matrix containing four independet error types. First, the absolute accuracy of the radar (3 dBZ; Danne et al. 1999) is considered by adding Gaussian noise with 3-dBZ standard deviation to the model dBZ. Also, the estimated LWP retrieval error is taken into account (section 2d). Errors due to the correction for hydrometeor attenuation can be interpreted as a measurement error and are also included in $\mathbf{S}_{1}$. The radar reflectivities calculated from the cloud model are attenuated according to (13). Then the correction for attenuation as described in section $3 b$ is applied to estimate the error. Since the optimal estimation equations do not explicitly include the forward model error due to the assumed $Z-\mathrm{LWC}$ relation, this error has been included in $\mathbf{S}_{1}$ in form of an equivalent error in $\mathrm{dBZ}$. The matrix $\mathbf{S}_{1}$ is calculated as a full matrix, that is, taking into account the correlations between all described errors. This will also smooth the LWC profile. Separate a priori profiles (Fig. 2) and covariance matrices were calculated for different vertical thicknesses of modeled clouds. This was done to create representative a priori LWC profiles, since the number of thin clouds in the model climatology dominates the number of thick clouds. Due to the nonlinearity of the problem $\mathbf{y}_{1}$ is written according to (10):

$$
\mathbf{y}_{1}=\mathbf{y}_{1, n}+\mathbf{K}_{1, n}\left(\mathbf{L W C}-\mathbf{L W C}_{1, n}\right) \text {. }
$$

Solving for $\mathbf{K}_{n} \mathbf{L W C}$ and taking the model climatology $\mathbf{y}_{2}$ (equal to $\mathbf{L} \mathbf{W} \mathbf{C}_{2}$ ) into account leads to the following [if (17) is used as an analogon]:

$$
\begin{aligned}
\widehat{\mathbf{L C C}}= & \left(\mathbf{S}_{2}^{-1}+\mathbf{K}_{1, n}^{\mathrm{T}} \mathbf{S}_{1}^{-1} \mathbf{K}_{1, n}\right)^{-1} \\
& \times\left\{\mathbf{K}_{1, n}^{\mathrm{T}} \mathbf{S}_{1}^{-1}\left(\mathbf{y}-\mathbf{y}_{1, n}+\mathbf{K}_{1, n} \mathbf{L} \mathbf{W} \mathbf{C}_{1, n}\right)\right. \\
& \left.\quad+\mathbf{S}_{2}^{-1} \mathbf{L} \mathbf{W} \mathbf{C}_{2}\right\} .
\end{aligned}
$$

This equation can now be iterated in $n$ taking $\widehat{\mathbf{L W C}}$ as $\mathbf{L} \mathbf{W C}_{1, n+1}$ until covergence is reached. The covergence criterion used here was $0.001 \mathrm{~g} \mathrm{~m}^{-3}$ in all layers. The final product is an LWC profile at all heights, where the measured or modeled radar reflectivity has a significant value above the radar detection limit.

\section{Retrieval accuracy}

In Fig. 5 the results obtained with the OE algorithm with the inclusion of the a priori profile (OE-ap) and without (OE-pure) are shown. Also an algorithm according to Frisch et al. (1998) is considered for comparison purpose. This algorithm is based on a powerlaw relation, which adjusts the coefficient $a$ according to the measured LWP weighted with the vertical sum of the $Z$ profile. The coefficient $b$ is held constant at a value of 2 assuming vertically constant droplet concentration and distribution width. This method is independent of radar calibration and DSD, provided that the sixth moment of the DSD can be related to the square of the third moment. The algorithm constrains the retrieved LWC profile exactly to LWP in a linear way.

In the following comparison, the GENE dataset is used to test the different methods. To simulate the real $\mathrm{dBZ}$ measurement a Gaussian noise distribution with 3$\mathrm{dBZ}$ standard deviation is added to the $\mathrm{dBZ}$ profile (Table 2). The obtained LWP value from the model is subject to noise corresponding to the error resulting from the LWP retrieval (section 2d). In the lowest $250 \mathrm{~m}$ above cloud base, both OE methods show a relative retrieval error of about $55 \%$ in comparison to $75 \%$ for the Frisch method. In the five heights above the lowest layer, the rms errors of the Frisch algorithm and the OEpure algorithm do not differ significantly, whereas the OE-ap method shows relative errors, which are smaller by about $10 \%$ to $15 \%$ (except at cloud top). The al- 
TABLE 2. GKSS 95-GHz radar characteristics while operating on 3 Mar 1999.

\begin{tabular}{lc}
\hline \hline Frequency & $95 \mathrm{GHz}$ \\
Peak power & $1.7 \mathrm{~kW}$ \\
Beamwidth & $0.17^{\circ}$ \\
Range resolution & $82.5 \mathrm{~m}$ \\
Antenna diameter & $1.2 \mathrm{~m}$ \\
Antenna gain & $60 \mathrm{~dB}$ \\
Dynamic range & $70 \mathrm{~dB}$ \\
Sensitivity & $-40 \mathrm{dBZ}$ in $1 \mathrm{~km}(1-\mathrm{s}$ averaging) \\
Transmit polarization & Horizontal or vertical \\
Receive polarization & Horizontal and vertical \\
Doppler processing & Pulse pair \\
Absolute dBZ accuracy & $\pm 3 \mathrm{dBZ}$ \\
\hline
\end{tabular}

gorithm bias is negative for both OE methods. This is an artifact of the minimization of logarithmized LWC values (Fig. 3). For the method of Frisch et al., the vertically integrated LWC bias is zero. But, as can be seen in Fig. 5c, the bias varies in a range of $0.06 \mathrm{~g} \mathrm{~m}^{-3}$ with an overestimation in the lower and an underestimation of LWC in the upper part of the cloud. This is due to the fact that total number concentration and distribution width are not constant with height in the modeled clouds. The OE-ap method has a larger negative bias than the OE-pure method because large overestimations of $\mathrm{LWC}$ at $\mathrm{dBZ}$ values larger than -30 are influenced most strongly by the inclusion of the a priori profile (Figs. 6a,b). For smaller dBZ values the a priori information has a smaller impact because the variance of the a priori profile is quite large. Still, the LWC rms is decreased notably in all $\mathrm{dBZ}$ ranges (Fig. 7) using the OE-ap algorithm. The rms error does not change significantly if the bias corrected version of the Z-LWC relation (section 2c) is used in the OE-ap algorithm. Only the bias of the $\mathrm{dBZ}$ class $\mathrm{A}, \mathrm{B}$, and $\mathrm{C}$ is closer to 0 . Due to the fact that the OE-ap algorithm is partially constrained to the LWP and the a priori profile, the bias correction is not as effective as in the simple Z-LWC relation.

To test the algorithm sensitivity towards the a priori profiles, which are derived from cloud-model data, the retrieval is performed with modified a priori profiles consisting of the original a priori profiles plus and minus their standard deviations (Fig. 2), respectively. The shift in positive and negative directions, respectively, of the a priori profile leads to a change in LWP bias of \pm 15 $\mathrm{g} \mathrm{m}^{-2}$ relative to the bias when the original a priori profile is used. The overall performance of the algorithm is not changed significantly due to the described modification.

\section{Measurements}

On 3 March 1999, combined measurements of the GKSS 95-GHz cloud radar and the 20-30-GHz channel radiometer were made at GKSS in Geesthacht, Germany. Radar and radiometer were located next to each other at a distance of about $5 \mathrm{~m}$. Both instruments were pointing vertically into the atmosphere. This section describes the instruments, the measurement situation, and the results of the new LWC algorithm.

\section{a. Cloud radar}

The radar used to derive the $Z$ profiles was MIRACLE (Microwave Radar for Cloud Layer Exploration) of GKSS, Germany. MIRACLE is described in detail by Danne et al. (1999). The system is a polarimetric Doppler radar operating at a frequency of $95 \mathrm{GHz}$ corresponding to a wavelength of $3.2 \mathrm{~mm}$. This frequency is located in a window region of the microwave spectrum, which has an atmospheric transmission of about $70 \%$ in the clear-sky case. Compared to typical X- or C-band radars, this W-band radar allows detection of much smaller droplet sizes with much less peak power. Also, the influence of ground clutter is almost negligible in comparison to longer wave radars due to MIRACLE's advanced antenna characteristics. The $Z$ profile is evaluated starting at $500 \mathrm{~m}$ above ground level to exclude most influences of the near field. The radar allows the derivation of Doppler velocity and Doppler spectrum width using the pulse pair processing method (Danne et al. 1999). An overview of the radar specifications is given in Table 2.
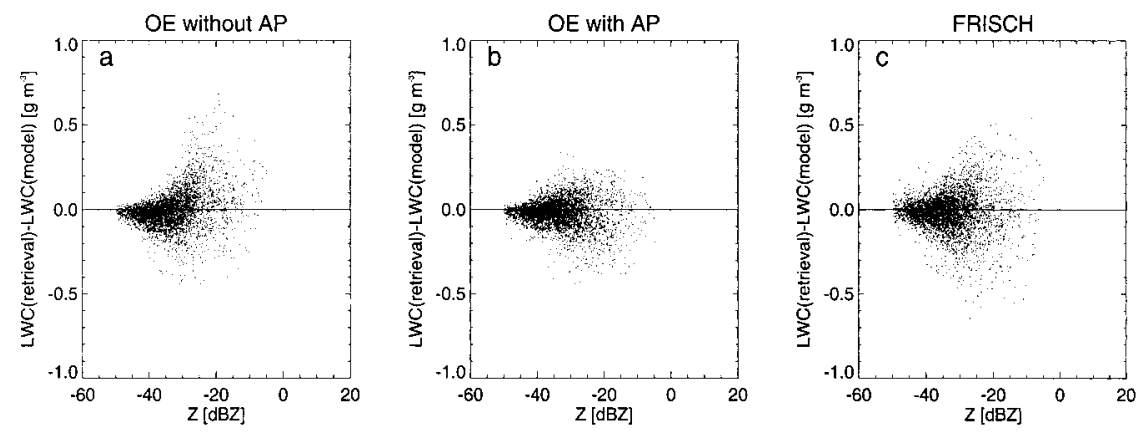

FIG. 6. Scatterplots of the three LWC retrievals in $625 \mathrm{~m}$ above cloud base. Left: Optimal estimation without inclusion of the a priori profile; center: optimal estimation with inclusion of the a priori profile; right: method according to Frisch et al. (1998). 

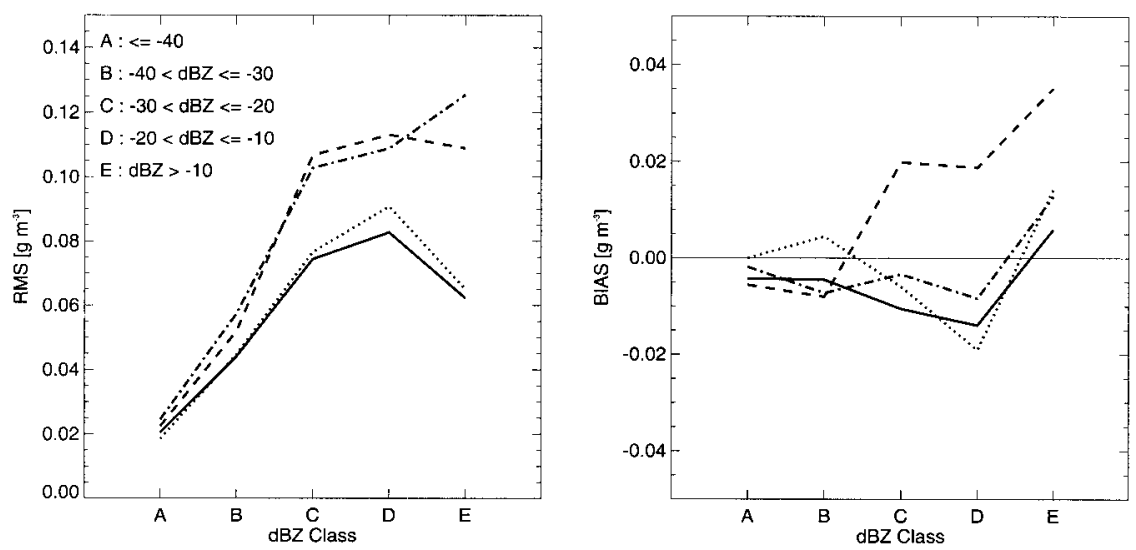

FIG. 7. The rms and bias errors of the LWC retrievals in dependence of the $\mathrm{dBZ}$ classes. Solid line: optimal estimation with a priori information; dashed line: optimal estimation without a priori information; dotted: optimal estimation with a priori information and $\mathrm{dBZ}$ bias correction; dasheddotted: method according to Frisch et al. (1998).

\section{b. Passive microwave radiometer}

The passive microwave radiometer measures atmospheric emission at 21.3, 23.8, and $31.7 \mathrm{GHz}$. It was built by Elektronik Centralen, Denmark, and was designed especially for highly accurate measurements during unattended operation. Calibration is performed applying the tipping curve method (Hogg et al. 1983). The radiometer is assumed to have a noise level of $1 \mathrm{~K}$ and a beamwidth of approximately $2^{\circ}$.

\section{c. Measurements}

Figure 8 shows the recorded time series of radar reflectivity and LWP during the afternoon of 3 March
1999 in Geesthacht, Germany. The synoptic situation was dominated by a low pressure region of about 975$\mathrm{hPa}$ central pressure over the British Isles. The corresponding cold front passed over the area of northern Germany in the night from 2 to 3 March 1999. After the front passage the surface layer cooled off significantly, giving rise to a temperature inversion in the upper part of the boundary layer. This corresponds quite well with an observed stratocumulus layer in the early afternoon ranging up to a quite sharp upper boundary of 2000 to $2500 \mathrm{~m}$ above ground level. After 14.5 UTC drizzle begins to dominate the reflectivity signal. At 14.6 UTC drizzle-sized drops seem to be falling out of the

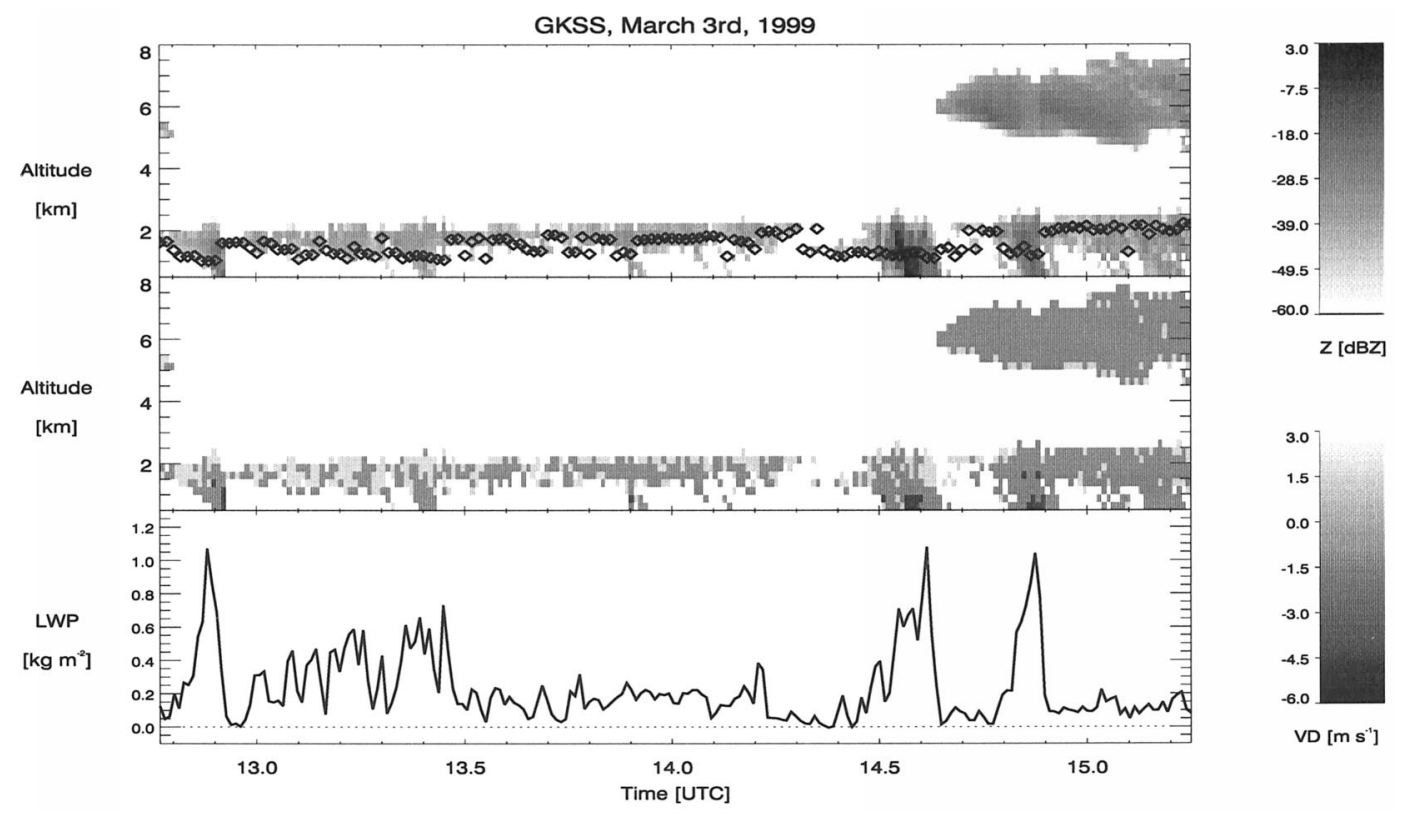

FIG. 8. Time series products of the collocated 20-30-GHz radiometer, the GKSS 95-GHz radar MIRACLE, and a Vaisala CT 25-K laser ceilometer. Top: radar reflectivity profiles with derived ceilometer cloud base heights (diamonds); center: Doppler velocity profiles; bottom: LWP derived from radiances at 20-30 GHz. 
cloud as indicated by Doppler velocities in the range from -4 to $-5 \mathrm{~m} \mathrm{~s}^{-1}$. Included in the upper part of Fig. 8 are cloud base heights (diamonds) derived from a Vaisala CT 25-K ceilometer (905-nm wavelength). The cloud-base height retrieval is based on the backscatter profile, which is proportional to the second moment of the DSD. This means that the backscatter coefficient is much more sensitive to small-cloud particles than drizzle, since normally cloud drops have number densities several orders of magnitude larger than drizzle drops. At 14.6 UTC, the ceilometer still detects the cloud base at about $1250 \mathrm{~m}$. This gives a further hint that larger drizzle drops below $1250 \mathrm{~m}$ are responsible for increasing the reflectivity signal up to $3 \mathrm{dBZ}$ in that region. After 14.9 UTC, radar cloud base and ceilometer cloud base again differ substantially. Doppler velocities indicate drizzle droplets falling out of the cloud layer. Retrieving liquid water with the described OE algorithm in these cases will be very difficult since drizzle does not necessarily carry considerable amounts of liquid water.

The LWC will thus only be retrieved for the time series until 14.5 UTC. Here radar and ceilometer cloud base, together with the Doppler velocities, indicate a statocumulus cloud composed of mostly smaller cloud droplets. The ground temperature during the time series was fairly constant at about $10^{\circ} \mathrm{C}$. No considerable amount of precipitation was recorded on the ground. Starting at about 14.6 UTC, the sky was overcast by clouds up to $4 \mathrm{~km}$ in thickness, ranging up to an altitude of $8 \mathrm{~km}$. It is probably correct to assume that these clouds consisted mostly of ice, which will not give a signal in the passive microwave measurement. Indeed, no considerable amount of LWP enhancement can be seen around 14.75 UTC, a phase where the stratocumulus layer is very thin. At 14.85 UTC a strong hydrometeor attenuation effect can clearly be seen in the ice cloud. The liquid water in the stratocumulus cloud, with $Z$ values up to $-18 \mathrm{dBZ}$, results in a decrease of $Z$ of about $10 \mathrm{dBZ}$ in the upper ice cloud due to attenuation.

\section{d. LWC profile retrieval}

First, the measured $Z$ profiles, which have a vertical resolution of $82.5 \mathrm{~m}$, were averaged to a vertical grid of $250 \mathrm{~m}$, and the radiometer measurements of TB were interpolated to the radar time steps. In cases of no radar reflectivity (e.g., 14.38 UTC), LWC is set to zero at all heights.

The bias-corrected OE-ap algorithm was applied to the first $1.7 \mathrm{~h}$ of measurements. After the first LWP peak at 12.9 UTC, reflectivities before and after 13.5 UTC do not differ very much. Until 13.5 UTC retrieved liquid water values range up to $0.7 \mathrm{~g} \mathrm{~m}^{-3}$ at cloud top, whereas LWC values barely exceed $0.3 \mathrm{~g} \mathrm{~m}^{-3}$ after 1350 UTC. This indicates the presence of relatively small drops in the beginning and larger drops later on. The larger LWP values until 13.5 UTC fit well to the Doppler velocities showing updrafts of up to $1.5 \mathrm{~m} \mathrm{~s}^{-1}$. This indicates a phase of cloud development. On the other hand, Doppler velocities around zero from 13.5 to 14.3 UTC and simultaneous values of lower LWP can be interpreted as a signal of cloud decay. As can be seen from the lower part of Fig. 9, the LWP resulting from the LWC algorithm is typically less than the radiometer retrieval. Particularly low reflectivity clouds $(\leq-40$ $\mathrm{dBZ})$ with considerable amounts of LWP $(\geq 0.1 \mathrm{~kg}$ $\mathrm{m}^{-2}$ )—for example, from 13.5 to 13.6 UTC-lead to underestimations, whereas clouds with reflectivities in the range of $-30 \mathrm{dBZ}$ are captured quite well in terms of LWP. This may be due to the fact that the vertical cloud extension is not fully resolved by the radar in low reflectivity regions, since this is also the region of the radar detection limit. Frequently, clouds are seen in the microwave signal or in the laser ceilometer backscattering signal and not in the radar signal. In this case the assumed a priori profile will be vertically too thin and constrain the algorithm toward a cloud containing less liquid water than the real cloud with a greater vertical extension. Further data will be evaluated in the near future to see if this effect is persistent. But as seen in section 5, the difference between radiometer-derived LWP and OE-derived LWC sum is only a limited test for the algorithm, since bias and rms errors in the different heights cannot be evaluated. This is only possible if in situ measurements are available.

\section{Conclusions and outlook}

Significant improvements retrieving LWC profiles can be achieved if a cloud radar and a microwave radiometer are combined. Model results indicate that if an a priori climatology is combined with $\mathrm{dBZ}$ and LWP measurements and their covariances are taken into account, further improvement of the LWC retrieval can be achieved in comparison to a method constraining the derived LWC profile exactly to the measured LWP. Relative errors in the range of $30 \%$ to $60 \%$ should be expected when using the described combination of instruments. In comparison, aircraft (in situ) measurements errors of the DSD, can also be in the range of $20 \%$ to $40 \%$ (French et al. 2000).

An important requirement the $\mathrm{OE}$ retrieval method should meet is a correct description of the variability of the modeled atmospheric states. In our case this is indicated by similar values of modeled and empiricaly measured coefficients $a$ and $b$. It would definitely be of advantage if large and representative climatological data sets of in situ measurements of different cloud DSDs were available. These could be used instead of the model statistics to derive an LWC algorithm.

One limitation to the algorithm is the occurrence of drizzle. Drizzle-sized drops can give the impression of a cloud reaching down close to the ground. This can lead to a false estimate of the liquid water distribution 


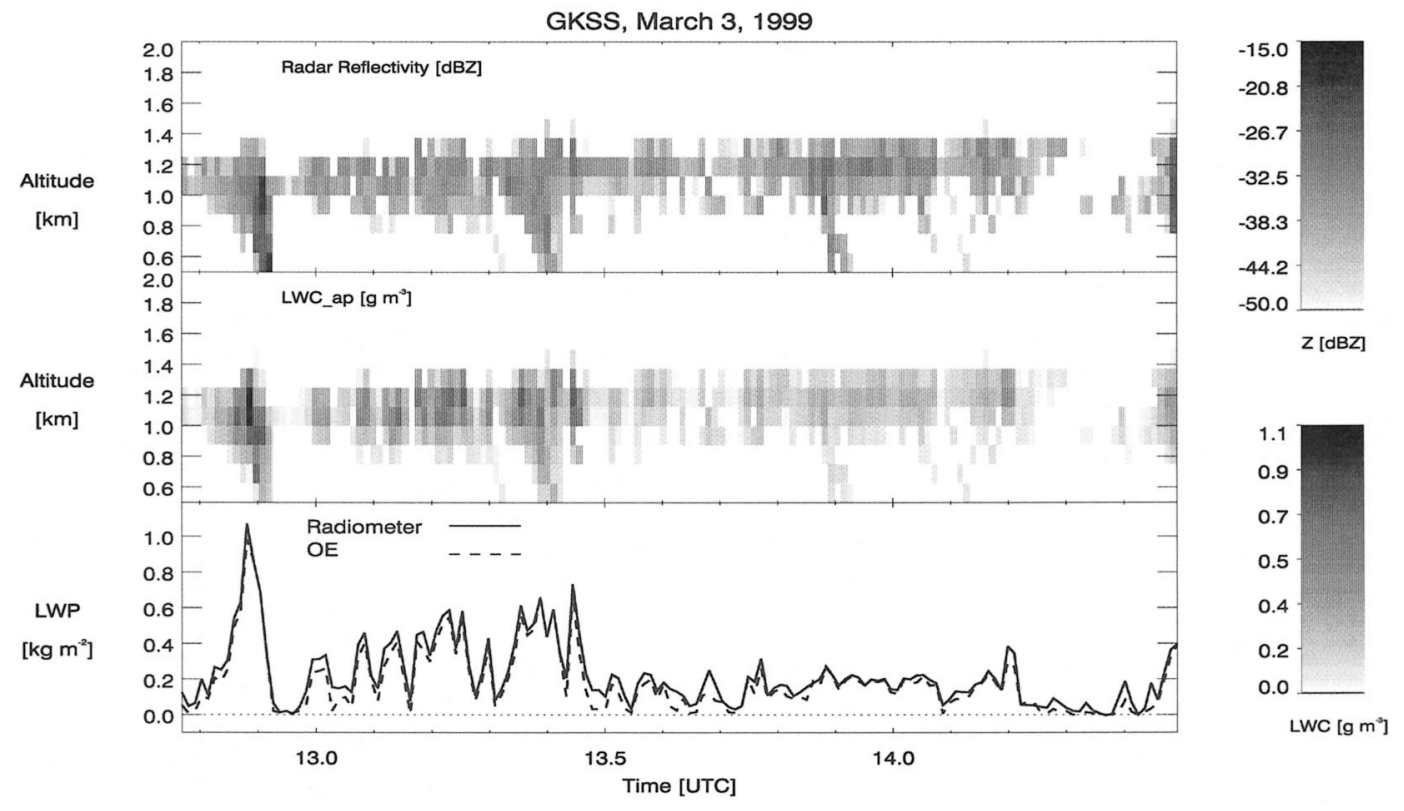

FIG. 9. Derived LWC time series. Top: Radar reflectivity profiles; center: LWC time series derived from the optimal estimation algorithm with inclusion of the a priori profile and the $\mathrm{dBZ}$ bias correction; bottom: radiometer-derived LWPs (solid) in comparison with the LWP resulting from the OE algorithm (dashed).

since the larger drizzle-sized drops lead to an overestimation of the LWC. As seen by regarding the Doppler velocities and laser ceilometer measurements, information about the occurrence of drizzle dominating the $\mathrm{dBZ}$ signal can be extracted. Methods have been proposed to solely calculate the drizzle liquid water content of a cloud (e.g., Frisch et al. 1995); however, the main problem in future will be not only to distinguish between nondrizzle and drizzle clouds, but to estimate the total liquid water content of both components in one cloud. A promising approach has been proposed by Czekala et al. (1999), who discriminate rain and cloud liquid by taking advantage of the polarization differences at low microwave frequencies due to nonspherical drop shapes.

A further uncertainty of the algorithm is the limitation to pure liquid water clouds. Pure ice cloud cases can be detected in our case since the radiometer frequencies used here do not respond to ice, but this instrument combination does not allow to distinguish between pure liquid and mixed phase clouds. Since very low ice water contents (e.g., $10^{-3} \mathrm{~g} \mathrm{~m}^{-3}$ ) can give rise to $Z$ values in the range of $-30 \mathrm{dBZ}$, it will be very important to develop methods to discriminate between cloud liquid and ice phase. Here, the utilization of the depolarization ratio of radar measurements or TB measurements at frequencies around $150 \mathrm{GHz}$ could be helpful.

Starting in the summer of 2000, ground-based measurement campaigns will be conducted at different sites of the BALTEX area within the EU project CLIWANET. CLIWA-NET (Cloud and Liquid Water-Network) is a network of 12 ground-based stations with microwave and infrared radiometers, cloud radars, laser ceil- ometers, and standard meteorological observations to determine large-scale variations of LWP fields. These fields will be used to calibrate satellite LWP retrievals and validate numerical weather forecast models. One major site will be at GKSS in Geesthacht, Germany. Here the 95-GHz cloud radar MIRACLE, a multichannel microwave radiometer, a laser ceilometer, and an infrared radiometer will be collocated. The new 22channel radiometer MICCY (Microwave Radiometer for Cloud Carthography) of the University of Bonn, Germany, will measure radiances in three spectral bands. Besides determining LWP more accurately than a 20 $30-\mathrm{GHz}$ combination, temperature and humidity information can be extracted (Crewell et al. 1999). Since microwave emission of liquid water increases with the frequency squared, assumptions can be made about the vertical distribution of LWC with a limited vertical resolution (Solheim et al. 1998). However, in contrast to the radar, the microwave signal is proportional to the third moment of the DSD meaning that even areas "contaminated" with drizzle will contribute with a signal proportional to the LWC, assuming the microwave signal is not saturated. Thus, the instrument combination of cloud radar and MICCY will be a very powerful tool in determining LWC profiles and DSD parameters. Currently an $\mathrm{OE}$ algorithm is being developed to combine these instruments. Additionally, aircraft measurements of cloud DSDs are planned during CLIWA-NET for algorithm validation.

Acknowledgments. The authors would like to thank O. Danne and M. Quante of the GKSS research center 
in Geesthacht, Germany, for providing the radar data, which was used in this work. We also thank the German Weather Service for providing the radiosonde data. We would like to acknowledge the work of D. Milferstädt from GKSS for supporting us technically at the combined radar-radiometer site. The two-channel radiometer was supplied by ESA/ESTEC and the laser ceilometer by the Institute for Marine Research (IfM) at the University of Kiel, Germany. This project was funded by the German Department of Research and Education under Grant 07 AK106/0.

\section{REFERENCES}

Clothiaux, E. E., M. A. Miller, B. A. Albrecht, T. P. Ackerman, J. Verlinde, D. M. Babb, R. M. Peters, and W. J. Syrett, 1995: An evaluation of a $94-\mathrm{GHz}$ radar for remote sensing of cloud properties. J. Atmos. Oceanic Technol., 12, 201-229.

Crewell, S., U. Löhnert, and C. Simmer, 1999: Remote sensing of liquid water profiles using microwave radiometry. Proc. Symp. Remote Sensing of Cloud Parameters: Retrieval and Validation, Delft, Netherlands, Delft University of Technology, 45-50.

Czekala, H., A. Thiele, A. Hornbostel, A. Schroth, and C. Simmer, 1999: Polarized microwave radiation from nonspherical cloud and precipitation models. Proc. Symp. Remote Sensing of Cloud Parameters: Retrieval and Validation, Delft, Netherlands, Delft University of Technology, 71-76.

Danne, O., M. Quante, D. Milferstädt, H. Lemke, and E. Raschke, 1999: Relationships between Doppler spectral moments within large-scale cirro- and altostratus cloud fields observed by a ground-based 95-GHz cloud radar. J. Appl. Meteor., 38, 175189.

Erkelens, J. S., H. W. J. Russchenberg, S. C. H. M. Jongen, and M. H. A. J. Herben, 1998: Combining radar and microwave radiometer for cloud liquid water retrieval. Proc. 28th European Microwave Conf., Vol. 2, Amsterdam, The Netherlands, IEEE/ MTT, 67-72.

Feingold, G., R. Boers, B. Stevens, and R. Cotton, 1997: A modeling study of the effect of drizzle on cloud optical depth and susceptibility. J. Geophys. Res., 102, 13 527-13 534.

Fox, N., and A. J. Illingworth, 1997a: The retrieval of stratocumulus cloud properties by ground-based cloud radar. J. Appl. Meteor., 36, 485-492.

— the detection of stratocumulus clouds. J. Appl. Meteor., 36, 676687.

French, J. R., G. Vali, and R. D. Kelly, 2000: Observations of microphysics pertaining to the development of drizzle in warm, shallow cumulus clouds. Quart. J. Roy. Meteor. Soc., 126, 415443.

Frisch, A. S., C. W. Fairall, and J. B. Snider, 1995: Measurement of stratus clouds and drizzle parameters in ASTEX with a Ka-band
Doppler radar and a microwave radiometer. J. Atmos. Sci., 52, 2788-2799.

— _ - G. Feingold, T. Utal, and J. B. Snider, 1998: On cloud radar and microwave radiometer measurements of stratus cloud liquid water profiles. J. Geophys. Res., 103, 23 195-23 197.

Hogg, D. C., F. O. Guiraud, J. B. Snider, M. T. Decker, and E. R. Westwater, 1983: A steerable dual-channel microwave radiometer for measurement of water vapor and liquid in the troposphere. J. Climate Appl. Meteor., 22, 789-806.

Issig, C., 1997: Ein spektrales Wolkenmodell mit integriertem Strahlungsübertragungsmodell zur Unterstützung von Niederschlagsalgorithmen. Ph.D. thesis, Meteorological Institute University of Bonn, 119 pp.

Karstens, U., C. Simmer, and E. Ruprecht, 1994: Remote sensing of cloud liquid water. Meteor. Atmos. Phys., 54, 157-171.

Liao, L., and K. Sassen, 1994: Investigation of relationships between Ka-Band radar reflectivity and ice liquid water contents. Atmos. Res., 34, 231-248.

Liebe, H. J., G. A. Hufford, and M. G. Cotton, 1993: Propagation modelling of moist air and suspended water/ice particles at frequencies below $1000 \mathrm{GHz}$. Proc. AGARD 52d Specialists Meeting of the Electromagnetic Wave Propagation Panel, Palma de Mallorca, Spain, AGARD, 3-1-3-10.

Ma, X., T. Schmit, and W. Smith, 1999: A nonlinear physical retrieval algorithm-Its application to the GOES-8/9 sounder. J. Appl. Meteor., 38, 501-513.

Peter, R., and N. Kämpfer, 1992: Radiometric determination of water vapor and liquid water and its validation with other techniques. J. Geophys. Res., 97, 18 173-18 183.

Pruppacher, H. R., and J. D. Klett, 1997: Microphysics of Clouds and Precipitation. Kluwer Academic, 953 pp.

Rodgers, C. D., 1976: Retrieval of atmospheric temperature and composition from remote measurements of thermal radiation. Rev. Geophys. Space Phys., 14, 609-624.

Sauvageot, H., and J. Omar, 1987: Radar reflectivity of cumulus clouds. J. Atmos. Oceanic Technol., 4, 264-272.

Simmer, C., 1994: Satellitenfernerkundung Hydrologischer Parameter der Atmosphäre mit Mikrowellen. Dr. Kovac, 313 pp.

Solheim, F., J. R. Godwin, E. R. Westwater, Y. Han, S. J. Keihm, K. Marsh, and R. Ware, 1998: Radiometric profiling of temperature, water vapor and cloud liquid water using various inversion methods. Radio Sci., 33, 393-404.

Ulaby, F. T., R. K. Moore, and A. D. Fung, 1981: Microwave Remote Sensing Active and Passive. Vol. 1. Artech House, 456 pp.

Vivekanandan, J., L. Li, L. Tsang, and C. Chan, 1997: Microwave radiometric technique to retrieve vapor liquid and ice: Part II. Joint studies of radiometer and radar in winter clouds. IEEE Trans. Geosci. Remote Sens., 35, 237-247.

von der Emde, K., and P. Kahlig, 1989: Comparison of the observed 19th July, 1981, Montana thunderstorm with results of a onedimensional cloud model using Kessler parameterized microphysics. Ann. Geophys., 7, 405-414.

Westwater, E. R., 1978: The accuracy of water vapor and cloud liquid water determination by dual-frequency ground-based microwave radiometry. Radio Sci., 13, 677-685. 